RAINER SILBERNAGL, Innsbruck

\title{
„... jedoch mit Willkühr nicht zu vermengen ist ..." Normen zur freien richterlichen Beweiswürdigung, zu Amtsbetrieb und Ermessen in den großen Zivilprozessordnungen Österreichs und Bayerns im 19. Jahrhundert
}

Legal Texts for the free consideration of evidence, ex officio proceedings and sole discretion in Austrian and Bavarian legal proceedings in the nineteenth century

The aim of this paper is to contribute (some) key facts concerning Austria's und Bavaria's history of litigation. Therefore, it intends to present the basic principles regarding Austrian and German civil legal proceedings: the free consideration of evidence for the judge recorded in $\S 272$ of the current Zivilprozessordnung. In this respect, emphasis will also be put on the ex officio proceedings and sole discretion.

The research work covers the nineteenth century, focusing on the codices of the Westgalizische Gerichtsordnung, on the Allgemeine Gerichtsordnung and on the Civilprozessordnung, including all the essential amendments. The analytical focus is on the Habsburgian monarchy of the nineteenth century. Finally, the free consideration of evidence is contrasted with the analogous system of the Bavarian und German law tradition in the Codex Juris Bavarici Judicarii and the Prozessordnung in bürgerlichen Rechtsstreitigkeiten für das Königreich Bayern.

Keywords: free consideration of evidence - ex officio proceedings - sole discretion history of litigation - codified laws of the early modern

Anlass zur Ausarbeitung dieses Beitrages war ein Vortrag auf der elften Tagung der Gesellschaft für Bayrische Rechtsgeschichte im Jahr 2014 in Innsbruck. Eine Verquickung des Themas zivilgerichtlicher Rechtsprechung zwischen den Ländern Tirol und Bayern bot sich daher aufgrund der in den Napoleonischen Kriegen erfolgten „bayrischen Herrschaft" in Tirol in den Jahren 1805-1809 an. ${ }^{1}$

Es sind mannigfaltige Faktoren, die den Richter davon überzeugen können, ein Vorbringen oder eine Tatsache für wahr zu halten oder nicht. Hinter Prozesseinrichtungen und der Auswahl

\footnotetext{
${ }^{1}$ FORCHER, Bayern-Tirol 116-129; LAICH, Zwei Jahrhunderte Justiz 51-61; SCHENNACH, Revolte in der Region 108, 188, 216-217.
}

der Inhaltsbestimmungen ist stets ein Ordnungsgedanke des Gesetzgebers ausgerichtet an der Umsetzung gewisser Ziele und Zwecke zu finden. ${ }^{2}$ Diese entziehen sich der normativen Ebene weitestgehend. Die Feststellungen, die das Gericht in seinem Urteil trifft, sind es, an die sich dann die Rechtsfolgen im Urteil knüpfen. Zentraler Punkt der folgenden Betrachtungen ist der "Grundsatz freier richterlicher Beweiswürdigung" in Zusammenschau mit den Vorschriften des Amtsbetriebes (ex officio) und dem Ermessen des Richters in den Prozessordnungen der Habsburgermonarchie mit Schwerpunkt auf dem Land Tirol sowie dem Königreich Bayern. Es ist die freie Beweiswürdigung, die dem Rich-

\footnotetext{
2 SPRUNG, Grundlagen 382.
} 
ter die nach menschlicher Logik zu bemessende Beurteilungskraft einzelner Beweismittel als Zentralpunkt des Erkenntnisverfahrens ermöglicht. Durch das prozessuale Werkzeug des Amtsbetriebes kann er auf die Geschwindigkeit des Prozesses z.B. durch die eigene Ausschreibung von Tagsatzungen, Beweiserhebungsterminen oder Bestimmung von Vertretern Einfluss üben, während das Vorherrschen der Verhandlungsmaxime den Richter in die alleinige Obhut der Parteien begibt. ${ }^{3}$ Der gesetzliche Ermessensspielraum gewährt ihm in prozessualen Notwendigkeiten wie bei der Bemessung der Länge von Fristen, der Bestimmung von Gebühren oder Festsetzung von Tagsatzungsabläufen Freiheiten bei Nebenschauplätzen des Gerichtsverfahrens, um nicht von der eigentlichen Streitsache abzulenken. Alle drei Institutionen sind mit der richterlichen Freiheit und Unabhängigkeit daher eng verzahnt.

Als Betrachtungszeitraum wurde das "lange 19. Jahrhundert" gewählt, dessen Beginn mit dem Jahr 1789, und dessen Ende mit dem Beginn des ersten Weltkrieges 1914, hier noch einmal prolongiert wird. Als Kontrastierung wird das (bisherige) Ende der Entwicklung, und zwar die heutigen Rechtsordnungen in Österreich $(\mathrm{ZPO})^{4}$ und Deutschland $(\mathrm{ZPO})$, den großen Prozessordnungen des 19. Jahrhunderts gegenübergestellt. Der Schwerpunkt der Betrachtungen verbleibt im österreichischen Recht.

\section{Gebundene oder freie Beweiswürdigung?}

Die Geschichte der freien Beweiswürdigung ist die Geschichte der Versuche, die individuelle Überzeugung des Richters, die an die Stelle der

\footnotetext{
${ }^{3}$ DAHLMANNS, Österreich 2739.

${ }^{4}$ Instruktiv zu den Grundzügen und der Entstehung der ZPO: SPRUNG, Zivilgerichtliches Verfahren 146151.
}

durch gesetzliche Beweisregeln gebundenen Beweiswürdigung treten sollte, durch mannigfaltige Kriterien zu objektivieren. ${ }^{5}$ Die Geschichte des Beweis- und des Würdigungsrechtes ist eine Geschichte des Prozesses überhaupt. ${ }^{6}$ In den römischen Prozessen der vorklassischen Periode gab es eine Reihe von Beweisregeln, die gegen Ende der Periode immer weniger wurden. In der klassischen Periode kann konstatiert werden, dass die Richter im Zivilprozess wesentlich freier in ihren Entscheidungen waren.? Die verschiedenen Beweismittel hatten verschiedene Wertigkeiten: Es gab die Parteienund Zeugenaussagen, den Augenschein, den Urkundenbeweis oder den Eid für die vorklassische Periode. Erstaunlich sind die prozessual ausmachbaren Grundsätze, nämlich Öffentlichkeit, Mündlichkeit, Unmittelbarkeit sowie der Verhandlungsgrundsatz, der sich in einer Herrschaft der Parteien über das Verfahren ausdrückte. Von einer Einheitlichkeit der Prozesse kann jedoch nicht gesprochen werden (Legisaktionenprozess, Formularprozess, Kognitionsprozess), ${ }^{8}$ es kann aber eine Entwicklung von festen Beweisregeln zu einem Mischsystem aus legalen Beweistheorien und richterlichem Ermessen verortet werden. ${ }^{9}$ In der nachklassischen Periode wurden aufgrund von Willkür, Missbrauch und Bestechlichkeit wieder mehr und mehr feste Beweisregeln eingeführt. ${ }^{10}$

Die nächste Periode wird im italienischkanonischen Prozess gesehen. Personell bezog sich die Verfahrensart vorerst auf Kleriker und auf Taten mit geistlichem Bezug, wobei das Verfahren aufgrund der tatsächlichen Wirkungsmacht, welche die Kirche auf das Leben im Ge-

\footnotetext{
${ }^{5}$ RECHBERGER, Maß für Maß 475f.; SCHNEIDER, Beweis und Beweiswürdigung Rz. 15f.

${ }^{6}$ WALter, Freie Beweiswürdigung 7f.

7 KASER, KNÜTEL, Römisches Privatrecht 462.

8 KASER, KNÜTEL, Römisches Privatrecht 434-438, 438446, 446-458.

${ }^{9}$ WALTER, Freie Beweiswürdigung 8-10.

${ }^{10}$ Huber, Beweismaß im Zivilprozeß $49 f$.
} 
nerellen hatte, zunehmend wohl auch auf weltliche Gerichte ausstrahlte. ${ }^{11}$ In Oberitalien entstand ein weltlich, germanisch-romanischer Mischprozess, der sich durch Durchnormierung einer legalen Beweistheorie hervortat und dem Richter, seiner Subjektivität und Willkür die Beweiswürdigung völlig entzog. ${ }^{12}$ Der Beweiswert einzelner Indizien wurde festgelegt und stark reglementiert. ${ }^{13}$ Die Prozessart steht dem römischen Prozess komplementär gegenüber und drängt die Freiheit des Richters in Form und Würdigung des Verfahrens nahezu gänzlich zurück. Zwar galt am Anfang des kanonischen Prozesses die freie Beweiswürdigung des Richters, doch die Furcht vor dessen Subjektivität bereitete einem breiten Spielfeld legaler Beweistheorien den Einzug. Über die Rezeption kamen diese Verfahrensregeln nach und nach ins heimische Recht. ${ }^{14}$

Die dritte relevante Verfahrensart ist der germanisch-altdeutsche Prozess, wie er sich im Laufe des Spätmittelalters darbot, um dann mit dem italienisch-kanonischen Prozess und dem römischen Prozessrecht in der Folge zusammenzuwirken. Der germanisch-altdeutsche Prozess entwickelte sich in gewisser Eigenständigkeit und kannte einige feste Beweisregeln (z.B. Eid, Zweikampf, Gottesurteil), die sich durch die sakrale und mythische Orientierung des Rechtsverständnisses verstehen lassen und bis zum Spätmittelalter etwas gelockert wurden. Von einer Einheitlichkeit der auf verschiedenen

\footnotetext{
11 WALtER, Freie Beweiswürdigung 22-39.

${ }^{12}$ Huber, Beweismaß im Zivilprozeß 52; SCHNEIDER, Beweis und Beweiswürdigung Rz. 16.

${ }^{13}$ GeIPEL, Handbuch der Beweiswürdigung Kap. 2 Rz. 6f: „[...] sogenannte Praesumptiones, also angebliche Gesetzmäßigkeiten und Erfahrungssätze wie z.B. dass jemand mehr um seine Angelegenheiten besorgt ist als um fremde. Die Praesumptiones ähneln somit dem heutigen Anscheins oder prima facie Beweis $[\ldots]^{\prime \prime}$.

${ }^{14}$ Huber, Beweismaß im Zivilprozeß 51f; GeIPEL, Handbuch der Beweiswürdigung Kap. 2 Rz. 20.
}

Volksrechten fußenden Verfahrensarten kann nicht gesprochen werden, wohl aber von tendenziellen Charakteristiken. Erwähnung verdient dabei die im germanischen Rechtsverständnis wurzelnde Sicht eines Gerichtsverfahrens. ${ }^{15}$ Im Unterschied zum römischen Prozess, in dem der Beklagte den Anspruch des Klägers nur leugnen und den Beweis desselben verlangen musste, um, wenn dieser nicht erbracht wurde, als Sieger aus dem Rechtsstreit hervorzugehen, bedeutete die Klage im germanischen Prozess mehr einen (moralischen) Vorwurf an den Beklagten, von dem er sich zu reinigen hatte. Die Klage war also eine persönliche Verletzung des Beklagten. Das Verfahren war auch nicht darauf ausgelegt, den Richter von der Wahrheit zu überzeugen, sondern nur darauf, den Beklagten von der Anschuldigung zu „reinigen“. Das Beweisurteil, welches nur darüber absprach, auf welchem Weg sich der Beklagte zu reinigen hatte, ließ demnach, da Eid, Zweikampf und Gottesurteil als „Beweismittel“ eine Beweisinterpretation in unserer gegenwärtigen Sichtweise nicht bewerkstelligten, keine Beweiswürdigung in unserem heutigen Verständnis zu. ${ }^{16}$ In der weiteren Entwicklung klagte der Behauptungseid unterstützt durch Eideshelfer den Beklagten an. Durch Leugnungseid und Urkunden sollten nun die Schöffen oder Geschworenen überzeugt werden. Ein Übergang zu freierer Beweiswürdigung wird in den Quellen dadurch markiert, dass die Parteien das Gericht fragten, „ob zur Sache genug vorgebracht und gewiesen sei?"

\footnotetext{
${ }^{15}$ WALter, Freie Beweiswürdigung 38-40.

${ }^{16}$ WALTER, Freie Beweiswürdigung 42f, vgl. auch SCHLOSSER, Spätmittelalterlicher Zivilprozess 338-384. 17 "Genug zu recht fürgebracht und geweist sei“ siehe die Nachweise bei SCHLOSSER, Spätmittelalterlicher Zivilprozess 386; WALTER, Freie Beweiswürdigung 49; Entwicklungsschritte vgl. auch BRINKMANN, Beweismaß im Zivilprozess aus rechtsvergleichender Sicht $21 f$.
} 
Im Spätmittelalter wurde der römischkanonische Prozess im Zuge der Reichskammergerichtsordnung 1495 im Heiligen Römischen Reich für den Zivilprozess eingeführt. Die Kollision zwischen italienisch-kanonischem und germanisch-altdeutschem Prozessrecht schlug zu Gunsten des ersteren aus. ${ }^{18}$ Das Landrecht passte sich dem Reichsrecht zeitlich versetzt an, sodass sohin vom gemeinen deutschen Prozess gesprochen werden kann. Bis zum Ende des 18. Jahrhunderts waren daher Beweistheorien über vollständigen und unvollständigen Beweis vorherrschend und gaben die Prozessregeln vor, wie ein unvollständiger Beweis zu einem vollständigen gemacht werden konnte. Wo die Lücke eines nicht vollständig erbrachten Beweises zu klaffen schien, konnte diese i.S. der vorherrschenden Beweiswerttheorien z.B. durch Eid vollständig gemacht werden. ${ }^{19}$ In der Zeit dieses reformierten Prozesses herrschte im Gebiet des (ehemaligen) Heiligen Römischen Reiches die Meinung vor, dass man es beamteten Richtern zur Vermeidung von Willkür nicht gestatten könne, nur nach ihrer freien Überzeugung zu urteilen. ${ }^{20}$ Dies gilt besonders für die im Folgenden betrachteten Prozessordnungen. Die Beweisregeln wurden am Ende dieser Entwicklung, zuerst in den „kleineren“ Verfahrensordnungen, nach und nach wieder zu Gunsten des Ermessens des Richters zurückgedrängt und mündeten in den heutigen Grundsatz der freien Beweiswürdigung. ${ }^{21}$

\footnotetext{
${ }^{18}$ Huber, Beweismaß im Zivilprozeß 52f.; Reichskammergerichtsordnung 1495; die Richter hatten zu geloben: „[...] nach des Reichs gemainen Rechten, auch nach redlichen, erbern und leidlichen Ordnungen, Statuten und Gewonhaiten der Fürstenthumb, Herrschaften und Gericht, die für sy pracht werden, [...] zu richten".

${ }^{19}$ WALTER, Freie Beweiswürdigung 76.

${ }^{20}$ GeIPEL, Handbuch der Beweiswürdigung Kap. 2 Rz. 9.

${ }^{21}$ GeIPEL, Handbuch der Beweiswürdigung Kap. 2 Rz. 8; WALTER, Freie Beweiswürdigung 76; SCHNEIDER, Beweis und Beweiswürdigung Rz. 17.
}

\section{Die Allgemeine Gerichtsordnung und die Westgalizische Gerichtsordnung}

Das 19. Jahrhundert ist in der Habsburgermonarchie geprägt von einer regen Gesetzgebungstätigkeit in nahezu allen (dogmatischen) Bereichen des Rechts (Stichworte: Gesetzesstaat, Gesamtstaatsidee). ${ }^{22}$ Als prominentestes Beispiel ist das ABGB (1812) zu nennen. ${ }^{23}$ Im Bereich dieser privatrechtlichen Kodifikation erweist sich als aufschlussreich, dass der Gesetzgeber die meisten novellierungsanfälligen bürgerlichrechtlichen Materien in die Policeygesetzgebung und ins spätere Verwaltungsrecht verschoben hatte und so im ABGB ein privatrechtlich stabiles Gerüst verblieb. ${ }^{24}$ Eine Tendenz, die in gewisser Weise auch in der AGO, WGGO und den daneben entstandenen summarischen Verfahrensordnungen erkennbar ist.

Die AGO vom 1. Mai 1781 brachte der Habsburgermonarchie auf dem Gebiet des Zivilprozesses die Rechtseinheit. Sie stellte eine knappe Zusammenfassung des gemeinrechtlichen Prozesses dar, wenn sie auch mit all dessen Nachteilen - wie Eventualmaxime, Schriftlichkeit des Verfahrens, gesetzliche Beweisregeln - belastet

\footnotetext{
22 SPRUNG, Entstehung und Weiterentwicklung des Außerstreitgesetzes 12 - 17.

${ }^{23}$ SCHLOSSER, Grundzüge der neueren Privatrechtsgeschichte 135-139; BRAUNEDER, Europäische Privatrechtsgeschichte 117, 121-123; WESENBERG, WESENER, Neuere deutsche Privatrechtsgeschichte 163-167, BALTL, KOCHER, Österreichische Rechtsgeschichte 185-188, BARTA, KOHLEGGER, StADLMAYER, Franz Gschnitzer Lesebuch 569-576; KROESCHELL, Deutsche Rechtsgeschichte 68f.; RAINER, Vom Probebetrieb zum 200-jährigen Bestehen 19-26; vgl. im Gesamten BARTA u.a., Kontinuität im Wandel oder DölEMEYER, MOHNHAUPT, 200 Jahre ABGB um beispielhaft zwei aus der Vielzahl Werke zu nennen, die aus Anlass des Festjahres 2011 erschienen.

${ }^{24}$ Instruktiv dazu SCHENNACH, ABGB - kodifikatorisches Umfeld.
} 
war. ${ }^{25}$ Im Bereich des formellen Privatrechtes folgt die WGGO der AGO 1781 nach etlichen Novellierungen nach und mündet die Kodifikationsentwicklung in die ZPO 1895.26 Die AGO hielt am schriftlichen, nicht öffentlichen Verfahren mit gesetzlichen Beweisregeln grundsätzlich fest. ${ }^{27}$ Weitere Kodifikationsbestrebungen zeitigten sich aufgrund der Umständlichkeit des Verfahrens ab 1816, deren bedeutsamstes Ergebnis das hier nicht behandelte Gesetz über das Bagatellverfahren vom 27. April 1837 war. ${ }^{28}$ Die prozessualen Kodifikationsbestrebungen fanden durch AGO bzw. WGGO also keinen Abschluss. Eher scheint sich die gesetzgeberische Gestaltungskraft den summarischen Verfahren zugewandt zu haben. ${ }^{29}$ Die AGO galt in weiten Teilen der Habsburgermonarchie. ${ }^{30}$ Da laut $\S 347$ der AGO die Gerichte nicht selbst über die Auslegung dieses Gesetzes entscheiden durften, hatten sie bei Hof anzufragen. So entstand eine Flut an Hofdekreten, Nachtragsverordnungen und Resolutionen, die die beabsichtigte Rechtseinheit zunehmend untergrub. ${ }^{31}$ Daher rührt die Ansicht, dass die AGO als rechtsvereinheitliche Zivilverfahrenskodifikation nicht ganz auf der Höhe der Zeit wahrgenommen wird, wohingegen die ihr nachfolgende ZPO an rechtschöpfender Modernität sogar einige Entwicklungsstufen des Zivilprozesses übersprang. ${ }^{32}$ Aus diesem Grund wurde eine Revision der AGO veranlasst und die sich daraus ergebende revisionierte Gerichtsordnung vorerst in Westgalizien

\footnotetext{
${ }^{25}$ MATSCHER, Entwicklung des zivilprozessualen Rechts Rz. 27.

${ }^{26}$ RGBl. 112/1895

27 OGRIS, Rechtsentwicklung 40-44.

${ }^{28}$ HUber, Beweismaß im Zivilprozeß 6; RASSE, Entwicklung der Juristenausbildung 40.

${ }^{29}$ DAHLMANNS, Österreich 2704, 2708 - 2714.

${ }^{30}$ LOSCHELDER, Österreichische Allgemeine Gerichtsordnung 80-83.

${ }^{31}$ SPERL, Lehrbuch der Bürgerlichen Rechtspflege 1617.

${ }^{32}$ DAhLMANNS, Österreich 2699, 2702.
}

in Kraft gesetzt. ${ }^{33}$ Unter dem Titel Westgalizische Gerichtsordnung vom 19. Dezember 1796 wurde diese Ordnung dann nach und nach in der gesamten Habsburgermonarchie publiziert. In Tirol und Vorarlberg waren die Ämter Vils, Ziller und Brixenthal bis 1. Oktober 1816 von der Geltung der WGGO ausgenommen. Ganzheitlich wurde die WGGO am 15. September 1814 in Kraft gesetzt. ${ }^{34}$ Insgesamt sollte es nach der Kundmachung der WGGO im Jahre 1797 noch mehr als fünfzig Jahre dauern, nämlich bis 1852, bis diese in der gesamten Habsburgermonarchie in Geltung war. ${ }^{35}$ Fragen über die Geltung und Auslegung der AGO (später WGGO) wurden per Hofdekret vom 10. Februar 1789 Nr. 897 beseitigt und angeordnet, dass das Beweisverfahren der WGGO derart erschöpfend sei, dass speziellere Rechte unbeachtet zu bleiben haben. ${ }^{36}$ Weitere Hofdekrete in diesem Sinne ergingen. ${ }^{37}$

Als Kontrapunkt der zivilprozessualen Entwicklungen wurde mit der für alle dem deutschen Bund angehörenden Teile der Habsburgermonarchie die Provisorische Strafprozeßordnung vom 17. Jänner 1850 in Kraft gesetzt, welche in Anlehnung an die Thüringische Strafprozessordnung und den dahinterstehenden französischen Code d'instruction criminelle von 1808 Staatsanwaltschaft, Anklageprozess, Geschworenengerichte und freie Beweiswürdigung in völligem Bruch mit dem (franziszäischen) Strafgesetzbuch von 1803 einführte. Die Strafprozessordnung 1853 wandte sich aber wiederum dem Inquisitionsprinzip zu, um die als Gesamt-

\footnotetext{
${ }^{33}$ MeNGER, System des österreichischen Zivilprozesses 62 .

${ }^{34}$ Menger, System des österreichischen Zivilprozesses 65 .

35 Niedermayr, Oberhummer, Peer, Ratsprotokolle der Obersten Justizstelle 29-32.

36 Vgl. zur Systematik z.B. STUBENRAUCH, Systematisches Handbuch 81-116, 119-188.

${ }^{37}$ MENGER, System des österreichischen Zivilprozesses 60 .
} 
heit betrachteten Verfahrensordnungen im Geiste von Rechtsgewissheit und -ordnung in ihrem josephinischen Ursprung $\mathrm{zu}$ erhalten. Erst mit der Strafprozessordnung vom 23. Mai 1873 wurde dieser Geist - aber immer noch 20 Jahre vor der entsprechenden Zivilverfahrenskodifikation - durchbrochen. ${ }^{38}$

Die Systematik der WGGO, welche aus 43 Kapiteln besteht und sich nur in ihrer Erweiterung und der Nummerierung der Paragraphen von der AGO unterscheidet, folgte bei der Beweisführung den legalen Beweistheorien. ${ }^{39}$ Die Regelungen der WGGO zur Beweisaufnahme künden bereits vom vorherrschenden Geist: Die Anwendung richterlicher Schlüsse und Logik wird insofern unterbunden, indem normiert wird, dass ausschließlich auf gesetzliche Vermutungen abzustellen ist und nur das für wahr zu halten ist, was ordentlich nach den strengen Beweisregeln als erwiesen gilt. ${ }^{40}$ Der unerbrachte Beweis oder das unbewiesene Gegenteil sind jedenfalls nicht für wahr zu halten. Dem allenfalls übereifrigen Richter wird untersagt, im Fall von Zweifeln noch eigenen Beweis aufzunehmen. ${ }^{41}$ Dies dürfte auch die Frage klären, warum in den meisten bisher gesichteten Akten in Verfahren nach der WGGO primär über Beweise und deren Führung gestritten wird und der

\footnotetext{
${ }^{38}$ DAHLMANNS, Österreich 2720.

39 DAHLMANNS, Österreich 2705.

${ }^{40}$ STUBENRAUCH, Systematisches Handbuch 161-163.

${ }^{41}$ Die folgenden $\S \S$ stammen aus der AGO.

$\S 170$. Wer ein Factum angeführt hat, er sey Kläger oder Beklagter, der ist schuldig, es zu erweisen, sonst ist bey Erledigung des Processes dasselbe, insoweit es von dem Gegentheile widersprochen worden ist, für wahr nicht zu halten.;

$\S 171$. Nur die in einem besonderen Gesetze gegründete Vermuthung kann von der Schuldigkeit des Beweises entheben.;

$\S 172$. Der Richter ist außer den Fällen, welche in dieser Gerichtsordung ausdrücklich vorgesehen sind, nicht befugt, weder den Parteyen einen Beweis, noch nach schon geführter Weisung einen mehreren Beweis aufzulegen [...].
}

Prozessgang schwerfällig und langwierig war. ${ }^{42}$ Die Grundausrichtung der AGO wird als ein Interessenstreit der Parteien gesehen, in dem der Richter aufgrund des starren gesetzlichen Korsetts nur ein geduldiger Mitspieler war. ${ }^{43}$

Regelungen zur Ermessensausübung durch den Richter sind in der WGGO wenige $\mathrm{zu}$ finden. Lediglich in neun der 620 Gesetzesstellen wird dem Richter expressis verbis Ermessen eingeräumt: Das Ermessen bestand im Concursverfahren hinsichtlich der Auswahl des Gläubigervertreters bei Stimmengleichheit (§95), in der Frage wieviel Glauben dem Vergleich von Urkundenschriften beizumessen ist (§ 209), ${ }^{44}$ in der Bemessung der Vergütung der Zeitversäumnis von Arbeitsleuten, wenn sie Zeugen waren (§ 239), inwieweit die Rückschiebung des Eides möglich sei (§ 278), 45 im Fristermessen (innerhalb eines gesetzten Rahmens) bei der gerichtlichen Versteigerung und Feilbietung von Liegenschaften $(\$ \S 435,460)$ oder in der Bestimmung des Kostenersatzes bei notwendigen Reisen

\footnotetext{
${ }^{42}$ MATSCHER, Entwicklung des zivilprozessualen Rechts 476-477; PRATOBEVERA, Ueber den Beweis durch Zeugen 338-345, ,[...] der Richter muß daher sowohl Form als auch Inhalt der Aussagen genau prüfen. [...] Das Gesetz wollte mit Recht für diese richterliche Prüfung und Abwägung (Anmerkung: der Vergleiche der Inhalte der formell korrekt ermittelten Beweise) keine Normen aufstellen, die auch hier nur in einigen Hauptzügen angedeutet werden können. Der Richter hat zum Behuf eines gründlichen Urtheils zu untersuchen 1) die Beschaffenheit der Aussage jedes einzelnen Zeugen für sich allein; 2) in Vergleichung mit den Aussagen anderer Zeugen; und 3) mit anderen Beweismitteln und Vermuthungen." 43 SPRUNG, Grundlagen 387.

${ }^{44} \S 209$. Wie viel Glauben die Vergleichung der Handschriften verdiene, wird nach Beschaffenheit der Umstände zu ermessen seyn.

${ }^{45} \S 278$. Wie weit in dem Falle, als der aufgetragene Eid nicht zurück geschoben werden könnte, der Gegentheil den Eid dennoch anzunehmen verbunden sey, hat der Richter nach dem Ermessen der Umstände zu beurtheilen; und hätte dieser auf Ablegung des Eides geurtheilet, so ist der Eid ohne alle Ausnahme abzulegen.
} 
(§ 538). Eine tatsächliche Freiheit räumt die Militärgerichtsbarkeit ein, die es dem Richter freistellte, zumindest die Verfahrensart zu wählen. ${ }^{46}$ Am deutlichsten wird die Furcht des obrigkeitlichen Gesetzgebers aber wohl in der Gesetzesstelle über die Bestimmung der Länge von Fristen: Ausdrücklich wird festgehalten, dass es sich beim bescheidenen Ermessen des Richters nicht um Willkür handelt. Das Gesetz alleine postuliert also schon, aus welchen Gründen es den Richtern einen derart strikten, von Beweistheorien durchdrungenen Gesetzesapparat vorgibt. ${ }^{47}$ In der freieren und eigenen Beurteilung des Richters verblieben weiters ebenso nur wenige Möglichkeiten. ${ }^{48}$ So konnte der Richter selbst bei mehreren bedenklichen Zeugen entscheiden, ob Sie den vollen Beweis erbringen, obwohl $\S 211$ und $\S 219$ Vorzeigebestimmungen für eine ge-

\footnotetext{
${ }^{46} \S 612$. Bey den Militär-Gerichten muß allgemein mündlich verfahren werden; doch hängt es von dem Einverständnisse beyder Theile, oder von dem klugen Ermessen des Richters ab, auch das schriftliche Verfahren fürzuwählen.

$47 \S 520$. Jede erste Frist erhält ihre Bestimmung einzig aus dem Gesetze; jede weitere Frist aus dem bescheidenen Ermessen des Richters, das jedoch mit Willkühr nicht zu vermengen ist, sondern sich auf die gezeigte und billig befundene Nothwendigkeit gründen muß; § 575. Die Richter sollen nach dem wahren und allgemeinen Verstande der Worte dieser Gerichtsordnung verfahren und sprechen, folglich einer von der klaren Vorschrift abweichenden Willkührlichkeit sich nie anmaßen. Nur dann, wenn ein Vorfall nicht in den Worten des Gesetzes entschieden wäre, ist derselbe aus der Anwendung der in gleichförmigen Fällen bestimmten Vorschrift zu entscheiden. Wird aber ein Richter die Streitsachen wider diese Ordnung verzögern, oder sonst die Parteyen durch üble Verwaltung seines Amtes beschweren, so hat er für allen Schaden zu haften. Eine solche Beschwerdeführung soll jedoch von der Partey nicht im Wege einer ordentlichen Klage, sondern bey dem oberen Richter angebracht, und durch diesen von Amts wegen genau untersuchet und entschieden werden.

48 NIPPEL, Erläuterung der Allgemeinen Gerichtsordnung 1, 3, 13.
}

bundene Beweiswürdigung darstellen. ${ }^{49}$ Diese Gesetzesbestimmungen können behutsam als ein kleines Reservat der freien Beweiswürdigung angesehen werden, da es dem entscheidenden Richter tatsächlich alleine überlassen blieb, die Glaubwürdigkeit (genauer: die Vollständigkeit) eines Beweismittels nach eigener innerer Überzeugung zu prüfen. ${ }^{50}$

Der Richter durfte bei Unterhaltssachen selbst entscheiden, wer Not litt (§ 482), die Schätzleute durften den Wert einer Sache frei schätzen (§§ 100, 273). Beim Schätzungseid konnte nach Eideszulassung und Aufkommen der Beweismittel der Richter den Betrag nach Billigkeit mäßigen (§ 292). Die Eidesformel war vom Richter selbst genau zu bestimmen (§ 281). Fristermessen wurde in Zustellungen, Tagsatzungen oder Repliken mehrfach eingeräumt. ${ }^{51}$

Die Bestimmungen über die Nullitätsbeschwerde ordnen dem übergeordneten Richter an, die Erfüllung der Zulassungsvoraussetzungen zu überprüfen, ehe er sich in die Sache einlässt und, sofern durch das Verhalten des untergeordneten

${ }^{49} \S 211$. Zu einem vollständigen Beweise, da dieser lediglich durch Zeugen geführt werden will, wird die einstimmige Aussage zweyer unbedenklicher Zeugen erfordert; wenn jedoch auch andere, obschon für sich allein nicht hinlängliche Beweismittel beygebracht worden sind, so kann die Aussage eines unbedenklichen oder auch eines oder mehrerer bedenklichen Zeugen den Beweis ergänzen. Nicht minder kann auch durch mehrere bedenkliche Zeugen ein vollständiger Beweis hergestellet werden. In solchen Fällen wird der Richter die Vollständigkeit des Beweises nach genauer Ueberlegung aller Umstände zu beurtheilen haben; §219. Wie viel Glauben einem bedenklichen Zeugen beyzumessen sey, hat der Richter nach genauer Ueberlegung aller Umstände $\mathrm{zu}$ beurtheilen.

${ }^{50}$ PrATOBEVERA, Ueber den Beweis durch Zeugen 259-263 „Fehlt es an zwei classischen Zeugen, so kann der reine legale Zeugenbeweis dennoch nach den Worten des Gesetzes vollständig durch mannigfaltige Combinationen, die dem richterlichen Ermessen anheim gestellt sind, gebildet werden".

${ }^{51} \S \S 445,30,20,31,36,41,66,85,142,168,314,400$, $476,435,578$ und 584 . 
Richters ein Schaden entstanden war und das Verzeichnis der Parteien darüber nicht einlangte, den zu vergütenden Betrag von Amts wegen auszumessen ( $\S \S 344,345$ und 346). Der übergeordnete Richter hatte aufgrund der ihm überreichten Beschwerdeschrift die Sache von Amts wegen $\mathrm{zu}$ untersuchen und darüber sobald wie möglich zu entscheiden (§352). Von Amts wegen sollte der Richter gemäß der WGGO grundsätzlich nie vorgehen. ${ }^{52}$ Dies stellt eingangs $\S 1$ fest, ausgenommen die Gesetze selbst würden den Richter hierzu verhalten.53 Dem Amtsbetrieb nach hatten die Richter aber bei Gefahr in Verzug den Vermögensverwalter einer Concursmasse, deren innere Beschaffenheit der Verschuldung sie zuvorderst zu untersuchen hatten (§ 97), zu bestellen ( 899 ) und dessen Verhalten und Belohnung eigenständig zu überwachen und zu mäßigen (§ 104, 155). Die §§ 224 und 225 regelten die an Zeugen jedenfalls zu stellenden Fragen und $\S 230$ das Verhalten des zur Einvernahme ersuchten Richters. Dies galt auch im Verfahren des Beweises zum ewigen Gedächtnis, einer Art Beweissicherungsvefahren (§ 254). Ebenso war bei einer Schadensschätzung der zu hoch geschätzte Schaden zu mäßigen (§ 290). Das Strafgericht hatte von Amts wegen vorzugehen (§ 309).54 Die Appellationsschriftsätze waren mitsamt dem Akt und allem dazugehörigen Material an den oberen Richter zu senden (§ 338). Über Zahlungsvorgänge bei einer öffentlichen Casse waren andere Behörden zu informieren $(\S \S 381,412)$. Zustellungen und Schuld-

\footnotetext{
${ }^{52}$ LOSCHELDER, Die österreichische Allgemeine Gerichtsordnung 93-96.

${ }^{53} \S 1$. Der Richter soll nur auf eine vorläufige Klage, und niemahls von Amts wegen verfahren, ausgenommen, da er hierzu durch die Gesetze angewiesen wird [...].

54 § 309. Glaubt Jemand erweisen zu können, dass sein Gegner einen falschen Eid abgelegt habe, so soll er dem Strafgerichte alle seine Beweismittel übergeben, und dieser nach Beschaffenheit der Umstände von Amts wegen die Untersuchung vornehmen.
}

nerverfolgungen im Konkurs- und Exekutionsverfahren waren gemäß $\S \S 476,479$ und 487 vorzunehmen. Insgesamt finden sich 21 Bestimmungen, die den Gang des Verfahrens von Amts wegen regeln. Der heutigen Verfahrenshilfe ähnliche Regelungen ergeben sich aus dem § 8 der Allgemeinen Taxordnung. Dieser regelte für Streitsachen in Ausübung des adeligen Richteramtes, in Grundbuchsgeschäften, in Bürgerrechts-, Gewerbs- und andern als taxbar oder nicht taxbar bestimmten politischen Gegenständen, in Kriminalvorfällen oder Lehenssachen, dass nachweislich armen Menschen sämtliche Taxen erlassen wurden. ${ }^{55}$ Diese Bestimmung ist in Verbindung mit der Bestimmung der WGGO in $\S 531 \mathrm{zu}$ lesen, nach der der Richter einem Armen von Amts wegen einen Vertreter zuzugeben hat. ${ }^{56}$

Inwieweit die gesetzlichen Regelungen des 26. Kapitels (§§ 356ff) über die gütliche Einigung allenfalls eine Flut an Klagen oder Streitigkeiten aus dem ordentlichen Verfahren herauszogen und die divergierenden Interessen zu einer Lösung zusammenbrachten, ist nach bisherigem Stand unbekannt. Dass das „ungestüme Einreden" des Richters auf die Parteien zur vergleichsweisen Lösung des Streites auch damals zu den zivilprozessualen Problemfeldern gehörte, wurde mehrfach wahrgenommen und versucht hintanzustellen. ${ }^{57}$ Allein aus der Tatsache, dass das Gesetz dies explizit vorsieht, lässt sich

\footnotetext{
${ }^{55}$ SEIDL, Allgemeine Taxnorme "§ 8 Dagegen sind jene, welche durch obrigkeitliches Zeugnis, oder sonstige Wege glaubwürdig ihre Mittellosigkeit darthun, gemäß der sie außer den unentbehrlichen nichts besitzen, von aller Taxe zu entheben [es folgt eine Regelung, die finanzstärkere Gegenpartei allenfalls in den Ersatz der Verfahrenskosten zu verfällen]".

${ }^{56} \S 531$. Dem einem Armen von Amts wegen zugegebenen Vertreter ist von dem Gegentheile, wenn er in den Ersatz der Gerichtsunkosten verfället wird, die Gebühr seines Verdienstes ebenfalls abzuführen.

${ }^{57}$ LOSCHELDER, Österreichische Allgemeine Gerichtsordnung 97.
} 
ableiten, dass das Anstreben einer außergerichtlichen Einigung ebenso anzustreben war.

Die WGGO baute also in kanonischer Rechtstradition auf einem System legaler Beweistheorien auf und hatte der Richter nur dort, wo das Gesetz seine Entscheidungsbefugnis überhaupt vorsah, fast ausschließlich anhand der gesetzlichen Normierungen zu einem Urteil zu kommen. $\mathrm{Zu}$ inhaltlichen Entscheidungen nach dem materiellen Recht kam es im Verfahren der WGGO oftmals nicht, sondern wurden Streitigkeiten nach formalen Fehlern beendet. Dies lässt die Vermutung entstehen, dass die rechtssuchende Bevölkerung wohl etwas unbillig davonkam. Die WGGO zielte jedoch darauf ab, die Fundierung des Urteils durch die Gründlichkeit formeller gesetzlicher Beweistheorien sicherzustellen. ${ }^{58}$ Sie schreibt dem Richter genau vor, wann er eine Tatsache für wahr zu halten hat, und lässt ihn Ermessen nur im kleinen gesetzlich vorgegebenen Rahmen üben. ${ }^{59}$ Prozessbeschleunigungen durch Ermessen und Amtsbetrieb finden sich ohne Stärkung der Richterposition nur in Randzonen des Prozesses.

\section{Der Codex Juris Bavarici}

Judiciarii und die

\section{Prozessordnung in bürgerlichen Rechtsstreitigkeiten für das Königreich Bayern}

In der Gesetzgebungstätigkeit wurde mit dem Codex Juris Bavarici Judiciarii (CIBI) im Bereich des formellen Rechtes der rechtliche Grundstein bereits im 18. Jahrhundert gelegt. ${ }^{60}$ Der CIBI hatte in der Zeit der eingangs erwähnten bayri-

\footnotetext{
${ }^{58}$ CANSTEIN, Civilprozessrecht 2, 279.

${ }^{9}$ Niedermayr, Oberhummer, PeER, Ratsprotokolle der Obersten Justizstelle 33.

${ }^{60}$ KobleR, Bayerische Kodifikationen 338; SCHLOSSER, Grundzüge der neueren Privatrechtsgeschichte 112116.
}

schen Herrschaft in Tirol Geltung. Verfahren, die bis 1809 nicht abgeschlossen waren, wurden nach dem CIBI weitergeführt. Seit 1848 war die Tendenz im bayrischen Königreich auf Verwirklichung der proklamierten staatsbürgerlichen Rechte und auf die Gewinnung einer neuen deutschen Einheit gerichtet. ${ }^{61}$ Die heutige Gerichtseinteilung geschah 1879 aufgrund des Reichs-Gerichtsverfassungsgesetzes vom 27. Januar 1877 und wurden dadurch 269 Amtsgerichte und 28 Landgerichte eingeführt. ${ }^{62}$ Die deutsche ZPO fand über die Reichsgesetzgebung im Zuge der Reservatrechte über das Reichsgesetz vom 30. Januar $1877^{63}$ Reichsgesetz Nr. 1167 Geltung im Königreich Bayern.

Der CIBI stellte im Amtsbetrieb ebenso wenige Vorschriften vor. Eingangs normiert Kapitel 5 $\S 11$ sogleich ein Verbot, dass der Richter in der Wahl der „Kontumazialwege dem gehorsamen Theile vorgreife". Falsche Anwälte, welche weder mit wahrer noch mutmaßlicher Vollmacht ausgestattet sind, sind von Amts wegen abzuweisen (Kap. 7 §7). Dem eifrigen Richter, der zwar von Amts wegen mehrere Zeugen vernehmen dürfte, wird insofern der Riegel vorgeschoben, als er hierzu nur in Angelegenheiten des öffentlichen Interesses ermächtigt ist. ${ }^{64}$ In Fristsachen der Gläubiger hat der Richter wiederum darauf hinzuwirken, dass eine Festsetzung

\footnotetext{
${ }^{61}$ KobleR, Bayern 335f.; KobLeR, Bayerische Kodifikationen $337 f$.

${ }^{62}$ Hiereth, Die bayerische Gerichts- und Verwaltungsorganisation 21, 28.

${ }^{63}$ Reichsgesetz Nr. 1167

${ }^{64}$ Die nachfolgenden Kapitel stammen aus dem CIBI: Kap. $10 \S 3$ Abs. 10; [...] 9) jetzt gedachter summarischer Beweis außer den im Kap. 3 § 3 genannten causis summariissimis ohne Bewilligung beider Partheien niemals Platz greifen, und obwohl endlich 10) dem Richter nach Gestalt der Umstände, nebst den vorgeschlagenen auch andere Zeugen von Amts wegen in summariissimo zu verhören nicht verwehrt ist, so soll doch dieses in bloßen Parthei- oder bürgerlichen Sachen, wo nicht das öffentliche Interesse miteinschlägt, nicht geschehen.
} 
der Frist von Amts wegen nicht notwendig wird. ${ }^{65}$ Im Konkursverfahren war es dem Richter gestattet, den Verwalter der Konkursmasse im Falle selbst zu bestimmen, aber es folgte auch wiederum die Mahnung sich nicht von Amts wegen mit Legitimationsfragen aufzuhalten (Kap. $19 \S 8$ Abs. 7; §18 Abs. 1). Überhaupt scheint der Amtsweg in 36 Gesetzestellen auf, die das Verfahren im Wesentlichen beschleunigen sollten. Völlig freie Entscheidungsmöglichkeiten für den Richter ergeben sich in der gesamten Textierung kaum.

Ermessensentscheidungen werden dem Richter bei der Vernehmung von Zeugen gegeben (Kap. 10 § 20 Abs. 1). Hier wird es seinem Ermessen völlig frei gestellt, welchen der widerstreitenden Zeugenaussagen er Glauben schenken will - solange es aber nur Nebenumstände betrifft. In der Hauptsache folgen dann die bereits im Rahmen der WGGO andiskutierten gewohnten Beweisregeln. ${ }^{66}$ Eine Erklärung dazu

\footnotetext{
${ }^{65}$ Kap. $18 \S 13$; [...] Sechstens, hat der Richter mit allem möglichen Fleiße dahin zu trachten, daß sich die Gläubiger eines Nachlasses selbst gutwillig auf Fristen und es mithin auf den Ausspruch von Amts wegen nicht ankommen lassen.

${ }^{66}$ Kap. 10 Von sich widersprechenden Zeugen und deren Beweiskraft: $\S 20$ Wenn die Zeugen selbst einander in ihrer Aussage widersprechen, so hat der Richter 1) zu sehen, ob dieser Widerspruch nur $\mathrm{Ne}-$ benumstände, oder die Hauptsache selbst betrifft. Im ersten Falle wird seinem Ermessen heimgestellet, ob, und in wie weit er ein solches widersprochenes Zeugniß sowohl auf der einen als der andern Seite für kräftig halten wolle, oder nicht, besonders wenn die Nebenumstände in solchen Kleinigkeiten und Sachen bestehen, welche man eben nicht allezeit so genau zu beobachten, oder auch leicht wieder aus dem Gedächtnisse zu lassen pflegt. Im andern Falle aber ist zuförderst 2) auf die Qualität, und alsdann auf die Anzahl der Zeugen zu sehen. Untüchtige Zeugen, soviel ihrer immer sind, schwächen die Aussage eines tüchtigen und exceptionsfreien Zeugen, wenn sie mit ihm in Widerspruch stehen, niemals soweit, dass seine Aussage nicht dennoch ihren sonst gewöhnlichen Effekt hätte. Wie weit ihm aber 3) zu glauben sey, wenn ihm zwar keine untüchtigen, aber doch
}

liefert Wiguläus Kreittmayr selbst, der sagt: „Bei mancher Gelegenheit beobachten wir alles genau, was in der Hauptsache dabei vorgehet, wir hören und sehen aber auch dabei vieles nur obenhin, machen uns eben keine starke Impression davon, und lassen es mithin auch leicht wieder aus dem Gedächtnis fahren und es beruhet demnach alles auf richterlichem Gutdünken". Aber selbst dieses Ermessen wird im Kommentar zum CIBI noch strukturiert, und werden Fallbeispiele für die Glaubwürdigkeit von Zeugenaussagen und Beweismittel angeführt, um dem Richter und Rechtsanwender eine Anleitung bei seiner "freien" Ermessensentscheidung zu geben. ${ }^{67}$ Eine Eigenart, die auch für die Kommentare der WGGO gilt.

Tatsächliche Freiheit, zumindest in der Wahl der Vorgangsweise, hatte der Richter bei Streitigkeiten wegen Kerbhölzern, also in Verfahren über

exceptionsmäßige Zeugen entgegenstehen, kommt auf richterliches Gutdünken an, dergestalt, dass in einem solchen Falle bald dem juramento suppletorio, bald dem purgatorio nach Gestalt der Dinge und Umstände Statt gegeben werden kann. Im Falle endlich 4) die einander widersprechenden Zeugen der Qualtität nach gleich und alle unmangelhaft sind, so kommt es auf die Zahl an, so dass 5) ein einzelner Zeuge den anderen widersprechenden Zeugen, und ebenso auch mehrere einzelne widersprechende Zeugen, soviel deren immer sind, gänzlich einander entkräften, folglich keiner von ihnen etwas beweisen kann. Wenn aber 6) ein einzelner Zeuge zwei anderen unter sich gleichförmigen widerspricht, so werden diese nur zur Hälfte entkräftet, und machen demnach einen halben Beweis. Drei oder mehr gleichförmige Zeugen hingegen wider einen einzigen, beweisen ganz und vollständig. 7) Wenn zwei oder mehr dergleichen stehen, so ist weder auf die Anzahl noch Würde derselben, sondern lediglich auf die Wahrscheinlichkeit der Aussagen zu sehen, mithin sind jene, welche am wahrscheinlichsten deponiren, oder wenn sie auch darin gleich sind, die zu Guten des beklagten Theils deponirenden Zeugen den anderen vorzuziehen.

${ }^{67}$ KREITTMAYR, Anmerkungen über den Codex juris Bavarici judiciarii 368-370. 
überschaubare Geldbeträge. ${ }^{68}$ In der Beurteilung der Echtheit von Abschriften ist ebenso ein freies Ermessen des Richters erkennbar, da dieser Beweise aufnehmen konnte, um die Abschrift für glaubhaft halten zu können. ${ }^{69}$ Die Beweisregeln bei Mutmaßungen räumen wiederum ein kleines richterliches Ermessen ein - verweisen vorsorglich aber dennoch auf das Gesetz. ${ }^{70}$ Bei Restitution (eigentlich: Unmöglichkeit der Restitution) der streitigen Sache obliegt es dem Richter, mittelmäßiges oder geringes Verschulden frei zu ermessen (Kap. $13 \S 4$ Abs. 2). Die Publikation des höherrichterlichen Appellationsbescheides liegt im Ermessen des höheren Richters (Kap. 15 § 11 Abs. 5). In Sachen der Kostenentschädigung kann der Richter, wenn der verlierende Teil Anlass zum Streit gehabt hatte, den Kostenersatz zu bemessen (Kap. 17 § 4 Abs. 3).

Eine tatsächliche freie Beweiswürdigung normiert hingegen ausdrücklich die Prozessordnung in bürgerlichen Rechtsstreitigkeiten für das Königreich Bayern vom 29. April 1869. Dort legt es Art. 345 dem Richter in die Hand, die Beweisführung nach freier Überzeugung $\mathrm{zu}$

\footnotetext{
${ }^{68}$ Kap. 11, § 11.). In Sachen der Privaturkunden, bei den bei Privatleuten üblichen kerbhölzern, steht es dem Richter frei zu entscheiden, welchem der Teile er nach Beschaffenheit der Beweise den Eid auferlegen möchte.

${ }^{69}$ Kap. 11 § 3 Abs. 3; § 4 Abs. 1; Kap. 11 Von Originalien und Abschriften: $\S 4$. Abschriften beweisen 1) für sich allein nichts, ausgenommen wenn sie sehr alt, und entweder im Archiv befindlich, oder wenigstens von einer bekannten Gerichts- oder Kanzleihand geschrieben, auch zugleich durch andere solche Behelfe mitunterstüzt sind, unter welchen Umständen erst in richterlichem Ermessen stehet, ob, und in wie weit dieselben Glauben verdienen [...].

${ }^{70}$ Kap. $12 \S 2$; [...] Welches alles jedoch 9) meistentheils auf richterliches Ermessen ankommt, wo im übrigen auch 10) bei gar zweifelhaften Vorfällen es allezeit vorzüglich bei dem bleibt, was das Gesetz hierinn ausdrückllich bestimmt, ungeachtet etwa verschiedene Präsumtionen dagegen vorwalten.
}

würdigen. ${ }^{71}$ Dies ist insofern von Interesse, als daneben auch feste Beweisregeln und Eidesformeln existieren, also eine Art "Mischverfahren“ konstruiert wird. Die Prozessordnung weist damit einen gewissen Sonderstatus als natürliches „Übergangsmodell“ zur freien Beweiswürdigung auf. Dazu hielt die zur Prozessordnung ergangene Kommentarliteratur euphorisch fest: „Das Gesetz ist bestrebt, überall der materiellen Wahrheit den Weg zu bahnen, und in diesem Bestreben will es vom Richter unterstützt sein“. ${ }^{72}$ Ihr beschränkter Geltungszeitraum von kaum acht Jahren im Übergang zur deutschen ZPO überließ ihr aber kaum Spielraum, dieses Wechselspiel zwischen Beweistheorie und freier Beweiswürdigung genauer zu entfalten. Für das Königreich Bayern ist mit der Prozessordnung in bürgerlichen Rechtsstreitigkeiten daher eine Übergangskodifikation zur deutschen ZPO auffindbar, welche, zumindest für des zivilistische Kernverfahren, für Österreich fehlt.

\section{Wesentliches zur heutigen freien Beweiswürdigung}

Für die französischen Geschworenengerichte wurde in Art. 342 Code d'instruction criminelle (1791) die sogenannte conviction intime ${ }^{73}$ festge-

\footnotetext{
${ }^{71}$ Der folgende Artikel stammt aus der Prozeßordnung in bürgerlichen Rechtsstreitigkeiten für das Königreich Bayern,

Art. 345: Bei Fällung des nach durchgeführtem Beweisverfahren $\mathrm{zu}$ erlassenden Urtheils hat das Gericht, wo das Gesetz nicht anders bestimmt, das Ergebniß der Beweisführung nach freier Ueberzeugung zu würdigen. Der Inhalt des Beweisurtheils ist hiebei nur soweit bindend, als in diesem etwa auf Eidesleistung erkannt ist. Hat sich durch das Beweisverfahren Beweis über mittelbar erhebliche Thatsachen (Art. 322) ergeben, so kann das Gericht diese berücksichtigen, auch wenn das Beweisanerbieten nicht darauf gerichtet war.

72 WernZ, Commentar zur Prozeßordnung 1, 356.

${ }^{73}$ DANIELS, Code d'instruction criminelle 161-163: „[...] Das Gesetz fordert von den Geschworenen keine
} 
legt, ein sich tief im Bewusstsein des jeweiligen Richters abspielender geheimnisvoller und unaussprechlicher Vorgang, der jeder Kontrolle entzogen war. Das Institut war nicht als reine Willkür gedacht, sondern richtete sich natürlich an ein hohes Ideal an Gewissenhaftigkeit und Rechtschaffenheit. ${ }^{74}$ Willhelm Endemann trat mit seiner Kampfschrift „Die Beweislehre des Civilprozesses" für die freie Beweiswürdigung in den "deutschen“ Nachfolgestaaten des Heiligen Römischen Reichs eine Diskussionswelle los. ${ }^{75}$ Sein Urteil über die Mitte des 19. Jahrhunderts vorgefundenen Beweissituationen in Deutschland war: „Der Zustand der Beweislehre in ihrer heutigen Gestalt kann für nichts weniger, als befriedigend gelten“. Den Deutschen Juristentag beschäftigte die Aussage Endemanns für drei Jahre mit intensiven Diskussionen, bis schließlich am 26. August 1863 eine Mehrheit den zaghaften Antrag formulierte: „Der Juristentag spricht sich - ohne damit bestimmten Vorschriften über die Zulässigkeit einzelner Beweismittel und über die Art ihrer Erhebung vorzugreifen, sowie mit Vorbehalt nöthiger Bestimmungen über den Beweis durch Urkunden und den Eid - für den Grundsatz aus, daß der Richter die Wahrheit der Thatsachen, soweit sie unter den Parteien streitig sind, nach freier Überzeugung zu prüfen habe“ ${ }^{\text {" }}{ }^{76}$ Dies legte einen weiteren Grundstein zur (Wieder-)Einführung der freien Beweiswürdigung, dem Ermessen des Richters und dessen prozessualen Handelns ex officio.

Rechenschafft über die Gründe, wodurch sie sich überzeugt gefunden haben; es schreibt ihnen keine Regeln vor, nach welchen sie vorzüglich beurtheilen solle, ob ein Beweis vollkommen und hinreichend sey: was es von ihnen fordert ist, daß sie in der Stille, und ganz in sich zurückgezogen, sich selbst befragen, und in dem Innersten ihres Gewissens erforschen [...]"; Code d'instruction criminelle 72-73.

${ }^{74}$ WALTER, Freie Beweiswürdigung 68f.

${ }^{75}$ ENDEMANN, Beweislehre des Civilprozesses VI-VIII, $622 \mathrm{f}$.

${ }^{76}$ WALTER, Freie Beweiswürdigung 83.
Die Beweiswürdigung hat heute in sich vollständig und geschlossen sowie widerspruchsfrei zu sein, sie darf keine Lücken aufweisen, nicht gegen gesicherte wissenschaftliche Erkenntnisse, Regeln der Logik und Erfahrungssätze verstoBen und soll das substantiierte Vorbringen der Parteien abwägen. Ihr edelster Zweck ist die Wahrheitsfindung. ${ }^{77}$ Beweiswürdigung ist die Beurteilung, ob ein bestimmter Sachverhalt durch apodiktische Schlüsse, bestehend aus einem Obersatz, Untersatz und Schlusssatz, als festgestellt, als zweifelhaft oder als widerlegt angesehen werden kann. ${ }^{78}$ Eine Anfechtung der Beweiswürdigung (Berufung im Punkt der Tatsachenfeststellungen) hat nach statistischen Erhebungen heute weder in Österreich noch in Deutschland große Aussicht auf Erfolg. ${ }^{79}$ Die Lösung der Tatsachenfrage ist dabei nicht weniger wichtig, oft sogar weitaus wichtiger als die Lösung der Rechtsfrage. ${ }^{80}$

Auszumachen sind in der heutigen zivilprozessualen Rechtslandschaft zwei große Meinungsblöcke: Die Anhänger der Überwiegenstheorie und die Vertreter der subjektiven Beweismaßtheorie. ${ }^{81}$ Die Frage des Beweismaßes ist praktisch die wichtigste Frage der Beweiswürdigung und legt als Rechtssatz fest, was zu prüfen ist, um einen Beweis als gelungen anzu-

${ }^{77}$ GEIPEL, Handbuch der Beweiswürdigung Kap. 1 Rz. 1; BALZER, Beweisaufnahme und Beweiswürdigung 17-20; BREHM, Bindung des Richters 52f; zur Kunst der Beweiswürdigung SCHNEIDER, Beweis und Beweiswürdigung Vf; BRINKMANN, Beweismaß im Zivilprozess 11; MusIELAK, STADLER, Grundfragen des Beweisrechtes Rz. 129.

${ }^{78}$ KRALIK, Beweiswürdigung 157; BALZER, Beweisaufnahme und Beweiswürdigung 17; HUBER, Beweismaß im Zivilprozeß 45.

${ }^{79}$ GeIPEL, Handbuch der Beweiswürdigung Kap. 1 Rz. 2.

${ }^{80}$ KRALIK, Beweiswürdigung 158; gegen die Unterschätzung der Tatfrage: SCHNEIDER, Beweis und Beweiswürdigung 1; TSCHADEK, Beweis 56-59.

${ }^{81}$ RECHBERGER, Maß für Maß 472. 
sehen. ${ }^{82}$ Einer der zentralen Grundsätze der österreichischen Zivilprozessordnung geltender Fassung ist das Institut der „freien Beweiswürdigung": der Richter hat unter sorgfältiger Berücksichtigung der Ergebnisse der gesamten Verhandlung - was einen Gesamtüberblick über die Geschehnisse im Gerichtssaal wie auch das Verhalten der im Prozess handelnden Personen beinhaltet - die durch die Beweismittel (Zeugen, Parteien, Urkunden, Lokalaugenschein und Sachverständigenbeweis) hervorgekommenen und herausgearbeiteten Beweisergebnisse nach freier Überzeugung zu beurteilen und $\mathrm{zu}$ würdigen, um festzustellen, ob eine tatsächliche Angabe für wahr zu halten ist oder nicht. ${ }^{83}$ Die Substantiierungspflicht der Parteien ist dabei der Motor des Verfahrens. ${ }^{84}$

Die österreichische Diskussion ist dabei bislang auf Sparflamme geführt worden. Die herrschende Meinung folgt der subjektiven Beweismaßtheorie. ${ }^{85}$ Die früheren gesetzlichen Beweisregeln geben also einerseits die Beweispflichtigkeit bzw. die Beweisintensität vor, und bestimmen, dass der Richter auf Grundlage der Beweisregeln den Beweis für wahr erachten muss. In der freien Würdigung allerdings entscheidet er selbst, ob in der dem Prozessgeschehen im Allgemeinen innewohnenden ex post Betrachtung der relevanten Ereignisse der von den Parteien behauptete Sachverhalt oder ein anderer Geschehensablauf für wahr (korrekter gesagt: wahrscheinlich) gehalten werden kann. ${ }^{86}$ Die Elemente der Beweiswürdigung verbinden sich dabei innerlich, ohne dass sie gesondert geprüft werden könnten. Die Überprüfung der erstgerichtlichen Beweiswürdigung obliegt dann dem

\footnotetext{
${ }^{82}$ BALZER, Beweisaufnahme und Beweiswürdigung 171; GEIPEL, Handbuch der Beweiswürdigung Kap. 6 Rz. 6.

${ }^{83}$ ReCHBERGER, SiMOTTA, Zivilprozessrecht 332-333.

${ }^{84}$ BREHM, Bindung des Richters 152.

${ }^{85}$ RECHBERGER, Maß für Maß 473.

${ }^{86}$ BRINKMANN, Beweismaß im Zivilprozess 23f.; DoLINAR, HOLZHAMMER, Zivilprozeßrecht 29-32.
}

Berufungsgericht und erstreckt sich im Wesentlichen darauf, ob sämtliche angebotenen Beweise aufgenommen und die aufgenommenen Beweise richtig beurteilt wurden ${ }^{87}$ und die (notwendige) Begründung ausreichend und logisch elaboriert wurde. ${ }^{88}$ Das Beweismaß zeigt sodann völlig abstrakt, wann der Beweis gelungen ist und die Beweiswürdigung, ob im konkreten Einzelfall eine Behauptung als wahr angesehen werden kann. Es handelt sich sohin um zwei voneinander $\mathrm{zu}$ trennende Positionen. Die Bestimmung des Beweismaßes ist eine Rechtsfrage, während die Beweiswürdigung eine Tatfrage ist. Der Begriff der Beweiswürdigung ist im Sprachgebrauch gängig geworden, korrekter sieht hier Andreas Geipel die Bezeichnung Beweis- und Verhandlungswürdigung, da nicht nur Beweise im engeren Sinn gewürdigt werden, sondern der gesamte Tatsachenstoff. ${ }^{89}$ Die der Beweiswürdigung innewohnende Begründungspflicht wird nach Ansicht der Wissenschaftler der geltenden rechtlichen Disziplin des Zivilprozessrechtes in der Praxis viel zu wenig ernst genommen..$^{90}$ Und dennoch gibt es eine rechtsfortgebildete Beschränkung dieser Entscheidungsfreiheit: das Regelbeweismaß engt die freie Beweiswürdigung - wenn auch unscharf - ein und haben dazu Judikatur und Lehre den Begriff des Regelbeweismaßes eingeführt. Die aktuellen Normierungen unterscheiden sich zwischen Österreich und Deutschland in eher geringem Maße.

\footnotetext{
${ }^{87}$ KRALIK, Beweiswürdigung 158-160.

88 DOLINAR, HOLZHAMMER, Zivilprozeßrecht 31f; RECHBERGER, SIMOTTA, Zivilprozessrecht Rz. 578f.

${ }^{89}$ GEIPEL, Handbuch der Beweiswürdigung Kap.6, Rz. 7, 9.

${ }^{90}$ Rechberger, Maß für Maß 475.
} 


\section{Freie Beweiswürdigung und Regelbeweismaß in den geltenden Zivilprozessordnungen}

Die Würdigung der Beweisergebnisse wird, obwohl sie in der Praxis eine zentrale Rolle einnimmt, im „Grundsatz der freien Beweiswürdigung" gesetzlich nur am Rande erwähnt. ${ }^{91}$ Ihre Herkunft im österreichischen Zivilverfahren liegt im Bagatellverfahren. ${ }^{92}$ Das Regelbeweismaß der $\mathrm{ZPO}^{93}$ ist die hohe Wahrscheinlichkeit. ${ }^{94}$

\footnotetext{
${ }^{91}$ BREHM, Bindung des Richters 1; TSCHADEK, Beweis $53-56$.

${ }^{92}$ Gesetz vom 27. April 1873, RGBl. 66/1873; SPERL, Lehrbuch der Bürgerlichen Rechtspflege 17-18; OBERHAMMER, $\mathrm{Zu}$ den Ursprüngen des Mahnverfahrens 286-300.
}

${ }^{93}$ RGBl. 112/1895; vgl. zur Entstehungsgeschichte MATSCHER, Entwicklung des zivilprozessualen Rechts 477-493; KODEK, MAYR, Zivilprozessrecht Rz. 28.

${ }^{94}$ Rechberger, Maß für Maß 484f; Huber, Beweismaß im Zivilprozeß 13; RS0110701: „Das Regelbeweismaß der ZPO ist die hohe und nicht eine an Sicherheit grenzenden Wahrscheinlichkeit; eine solche ist nur in den Fällen eines erhöhten Regelbeweismaßes erforderlich.“; OGH 30. 03. 19993 Ob 314/97s, Beisatz: „In den Tatsachenfeststellungen eines Urteils muss aber eindeutig zum Ausdruck kommen, ob ein bestimmter, für die Entscheidung wesentlicher Umstand festgestellt wird oder dass eine solche Feststellung nicht möglich ist, weil der Umstand nicht mit dieser hohen Wahrscheinlichkeit als erwiesen angenommen werden kann.“; OGH 22. 10. 200210 Ob 98/02p, Beisatz: „Eine „an Sicherheit grenzende Wahrscheinlichkeit" reicht für die Erbringung eines Beweises jedenfalls aus" (SZ 23/26; SZ 21/25; Fasching, LB2 Rz 815).“; OGH 17. 11. 2004, 7 Ob 260/04t, Beisatz: „Hohe Wahrscheinlichkeit stellt keine objektive Größe dar. Einem solchen Regelbeweismaß wohnt eine gewisse Bandbreite inne, sodass es sowohl von den objektiven Umständen des Anlassfalles als auch von der subjektiven Einschätzung des Richters abhängt, wann er diese "hohe" Wahrscheinlichkeit als gegeben sieht."; OGH 25. 06. 2009, 2 Ob 39/09p: „Das Regelbeweismaß der ZPO ist die hohe und nicht eine an Sicherheit grenzenden Wahrscheinlichkeit."; OGH 22.06. 2011 2 Ob 97/11w, Beisatz: „Das Regelbeweismaß der ZPO
Der Jurist hat sich bei der Bestimmung des Begriffs der Wahrscheinlichkeit an der Wahrscheinlichkeitslehre der Naturwissenschaften $\mathrm{zu}$ orientieren. ${ }^{95}$ Verankert ist die freie Beweiswürdigung - ganz im Zeichen der beabsichtigten Praktikabiltät und Handhabbarkeit des Verfassers der $\mathrm{ZPO}^{96}$ - in $\S 272 .{ }^{97}$

Ein wenig umfangreicher gestaltet sich die Situation der aktuellen Judikatur in Deutschland. ${ }^{98}$ Die deutsche Zivilprozessordnung wurde durch das Reichsgesetz Nr. $1167^{99}$ in Deutschland im

ist nicht die (bloß) überwiegende, sondern die hohe Wahrscheinlichkeit".

${ }^{95}$ MusielaK, STADLER Grundfragen des Beweisrechtes Rz. 134.

${ }^{96}$ DAHLMANNS, Österreich 2738.

${ }^{97}$ Huber, Beweismaß im Zivilprozeß 2; Geltende Fassung, seit Kundmachung in RGBl. 113/1895 unverändert:

„§ $272 \mathrm{ZPO}$

(1) Das Gericht hat, soferne in diesem Gesetze nicht etwas anderes bestimmt ist, unter sorgfältiger Berücksichtigung der Ergebnisse der gesammten Verhandlung und Beweisführung nach freier Überzeugung zu beurtheilen, ob eine thatsächliche Angabe für wahr zu halten sei oder nicht.

(2) Es hat insbesondere in gleicher Weise zu entscheiden, welchen Einfluss es auf die Beurtheilung des Falles hat, wenn eine Partei die Beantwortung von Fragen verweigert, welche durch den Vorsitzenden oder mit dessen oder des Senates Zustimmung an sie gestellt werden.

(3) Die Umstände und Erwägungen, welche für die Überzeugung des Gerichtes maßgebend waren, sind in der Begründung der Entscheidung anzugeben".

98 Geltende Fassung „§286 Freie Beweiswürdigung: (1) Das Gericht hat unter Berücksichtigung des gesamten Inhalts der Verhandlungen und des Ergebnisses einer etwaigen Beweisaufnahme nach freier Überzeugung zu entscheiden, ob eine tatsächliche Behauptung für wahr oder für nicht wahr zu erachten sei. In dem Urteil sind die Gründe anzugeben, die für die richterliche Überzeugung leitend gewesen sind. (2) An gesetzliche Beweisregeln ist das Gericht nur in den durch dieses Gesetz bezeichneten Fällen gebunden".

${ }^{99}$ Gesetz betreffend die Einführung der Civilprozeßordnung vom 30. Januar 1877. 
Rahmen der Reichsgesetzgebung eingeführt. ${ }^{100}$ Die freie richterliche Beweiswürdigung war darin im Wesentlichen - wenn vorerst auch noch an unterschiedlichen Stellen - festgeschrieben. Das reichsgerichtliche Erkenntnis ${ }^{101}$ aus 1884 postuliert: „Deswegen gilt im praktischen Leben der hohe Grad von Wahrscheinlichkeit, welcher bei möglichst erschöpfender und gewissenhafter Anwendung der vorhandenen Mittel und Erkenntnisse entsteht, als Wahrheit und Bewusstsein des Erkennenden von dem Vorliegen einer so ermittelten hohen Wahrscheinlichkeit, als die Überzeugung von der Wahrheit" “102, wobei einige höchstrichterliche Abstufungen dieses Rechtsatzes erfolgten. ${ }^{103}$ Der Bundesge-

${ }^{100}$ Vgl. MitTeis, LieBerich, Deutsches Privatrecht 22. ${ }^{101}$ RG, 14. 1. 1885 - I 408/84 veröffentlicht in RGZ 15, S. 228, 339; dazu insbesondere EVERS, Begriff und Bedeutung der Wahrscheinlichkeit für die richterliche Beweiswürdigung 3-8; vgl insbesondere die Versuche in MusielaK, STADLER, Grundfragen des Beweisrechtes Rz. 146 die Wahrheitsüberzeugung des Richters auf ein Fundament zu stellen.

102 BRINKMANN, Beweismaß im Zivilprozess 41f; HUBER, Beweismaß im Zivilprozeß 56ff., 121ff.; GEIPEL, Handbuch der Beweiswürdigung Kap.6 Rz. 86; jüngst wieder zitiert: OLG München vom 06.07.2012 10 U 3111/11 „Diese Überzeugung des Senats erfordert keine - ohnehin nicht erreichbare (vgl. RGZ 15, 338 [339]; Senat NZV 2006, 261; 28.07.2006 - 10 U 1684/06 [Juris], stRspr., zuletzt 11.06.2010 - $10 \mathrm{U}$ 2282/10 [Juris] = NJW-Spezial 2010, 489 f. mit zust. Anm. Heß/Burmann ) - absolute oder unumstößliche Gewissheit und auch keine „an Sicherheit grenzende Wahrscheinlichkeit“, sondern nur einen für das praktische Leben brauchbaren Grad von Gewissheit, der Zweifeln Schweigen gebietet (grundlegend BGHZ 53, 245 [256] = NJW 1970, 946, stRspr., zuletzt NJW 2008, 2845 und NJW-RR 2008, 1380; OLG Frankfurt/Main ZfS 2008, 264, 265; Senat NZV 2003, 474 [Revision vom BGH durch 01.04.2003 - VI ZR 156/02 - nicht angenommen]; NZV 2006, 261; 28.07.2006 - $10 \mathrm{U}$ 1684/06 [Juris], stRspr., zuletzt 11.06.2010 - $10 \mathrm{U}$ 2282/10 [Juris] = NJW-Spezial 2010, 489 f. m. zust. Anm. Heß/Burmann und NJW 2011, 396, 397 = VersR 2011, 549 ff. m. zust. Anm. Hoffmann ); v. 21.10.2011 10 U 1995/11 [Juris])“.

${ }^{103}$ GEIPEL, Handbuch der Beweiswürdigung Kap. 6 Rz. 97f: „RGSt 15 vom 20.12. 1886, 151-153 „Anast- richtshof entschied in BGHZ 53, S. 255ff „Denn nach [nunmehr geltendem] § 286 ZPO muss der Richter auf Grund der Beweisaufnahme entscheiden, ob er eine Behauptung für wahr oder unwahr hält, er darf also gerade nicht mit einer bloßen Wahrscheinlichkeit beruhigen. Im Übrigen stellt § 286 ZPO nur darauf ab, ob der Richter selbst die Überzeugung von der Wahrheit einer Behauptung gewonnen hat. Diese persönliche Gewissheit ist für die Entscheidung notwendig, und allein der Tatrichter hat ohne Bindung an gesetzliche Beweisregeln und nur seinem Gewissen unterworfen die Entscheidung zu treffen, ob er die an sich möglichen Zweifel überwinden und sich von einem bestimmten Sachverhalt als wahr überzeugen kann. Eine von allen Zweifeln freie Überzeugung setzt das Gesetz dabei nicht voraus. Auf diese eigene Überzeugung des entscheidenden Richters kommt es an, auch wenn andere zweifeln oder eine andere Auffassung haben würden. Der Richter darf und muss sich aber in tatsächlich zweifelhaften Fällen mit einem für das praktische Leben brauchbaren Grad von Gewissheit begnügen, der den Zweifeln Schweigen gebietet, ohne sie völlig auszuschließen". ${ }^{104}$

In Abstufungen ist also entscheidend, dass der verhandlungsführende Richter zur persönlichen Überzeugung kommt, womit naturgemäß eine starke subjektive Komponente in die Tatfrage einfließt, aber es eben doch noch auf die Wahrscheinlichkeit ankommt. ${ }^{105}$ Ein insgesamt einheitliches Bild der derzeitigen Judikatur gibt es nicht, da an die Begriffe der Wahrscheinlichkeit

hasiaentscheidung. Liegt [...] ein an Gewissheit angrenzender Grad von Wahrscheinlichkeit vor, [...] so ist damit der Beweis geliefert." oder auch WALTER, Freie Beweiswürdigung 103; EVERS, Begriff und Bedeutung der Wahrscheinlichkeit 7f; MUSIELAK, STADLER, Grundfragen des Beweisrechtes Rz. 130f.

${ }^{104}$ GEIPEL, Handbuch der Beweiswürdigung Kap. 6, Rz. 107-131.

105 SCHNEIDER, Beweis und Beweiswürdigung Rz. 3649. 
und des Beweismaßes doch mehr oder weniger schwache subjektive Überzeugungskomponenten an den entscheidenden Zivilrichter gestellt werden. ${ }^{106}$

Die Begründungspflicht der Beweiswürdigung des Richters wurde in $\S 286$ Abs. 1 Satz 2 der deutschen ZPO (1877) eingeführt und im Folgenden der Regelung des § 272 Abs. 3 der österreichischen ZPO (1898) nachempfunden. ${ }^{107}$ Die Diskussion über die freie Beweiswürdigung stieß auf Gegner, gegen die als schlagkräftigstes Argument die Begründungspflicht ins Feld geführt wurde, da der Richter sich nicht mit formelhaften Phrasen aus der Affäre ziehen konnte, sondern seine Gedankengänge offenzulegen hatte. ${ }^{108}$ Die Begründungspflicht und somit die Nachvollziehbarkeit des Gedankenganges zur Beweiswürdigung stellen in Verbindung mit der freien Überzeugung den Kernbereich heutigen Rechtsdenkens über den gelungenen Beweis dar.

\section{Zusammenfassung}

Bei der gängigen Abwertung gesetzlicher Beweisregeln sollte nicht verkannt werden, dass es sich um keine willkürlichen Regeln handelt, sondern diese Regeln die Ergebnisse psychologischer Einsichten von Jahrhunderten sind. ${ }^{109}$ Eine Pauschalverurteilung der mit legalen Beweistheorien arbeitenden Gerichtsordnungen

\footnotetext{
${ }^{106} \mathrm{Zu}$ den Meinungen vgl Evers, Begriff und Bedeutung der Wahrscheinlichkeit 12-39; HuBER, Beweismaß im Zivilprozeß 67-88; GEIPEL, Handbuch der Beweiswürdigung Kap. 6, Rz. 105, 109, 115, 124, 129, 135-156, der hier unter anderem die Worte uneinheitlich, verwirrend, unprognostizierbar verwendet und den wohl berechtigten Vorwurf erhebt, der BGH arbeite hier mit verschiedenen nicht zusammenpassenden Beweiswürdigungstheorien.

107 RECHBERGER, Maß für Maß 475.

${ }^{108}$ Huber, Beweismaß im Zivilprozeß 8f.

${ }^{109}$ GEIPEL, Handbuch der Beweiswürdigung Kap. 2, Rz. 18.
}

des 19. Jahrhunderts als Kinder ihrer Zeit wird durch das sicherere rechtsstaatliche Trittbrett der Gegenwart natürlich erleichtert. ${ }^{110}$ Die gesetzgeberische Leistung der AGO und WGGO muss eher darin begründet sein, dass sie dem riesigen kulturell und sprachlich divergierenden Länderkonglomerat der Habsburgermonarchie eine gewisse Rechtseinheit brachte. Die starren Prozessregeln der WGGO sollten jedem Richter, ${ }^{111}$ gleich welcher der verschiedenen Sprachoder Kulturgruppen er angehörte, ein Verfahren nach den gleichen Grundsätzen ermöglichen. Umgekehrt bedeutet dies, dass der Staatsbürger in jedem Kronland eine nach legalen Beweistheorien gleichgeschaltete, starr arbeitende Justiz vorfand. In Bayern herrschte dagegen kulturelle und sprachliche Einheit vor.

Die Sentenz über die AGO und die WGGO muss sohin bleiben, dass die Beweiswürdigung des Richters strikt durch den gesetzlichen Wert, der den Beweismitteln beigemessen wurde, gebunden war. ${ }^{112}$ Die Starrheit dieses Systems beschränkte ein Eingehen des Richters auf speziellere Prozesskonstellationen. ${ }^{113}$ Es zeigen sich zwar kleine Freiheiten im Rahmen der Prozessleitungsbefugnis, insbesondere was die Regelung der Prozessdauer oder gewisse Kostenentscheidungen betrifft. Das Gesetz bleibt aber sperrig, jedoch erschließt sich dem Leser eine gewisse Systematik, auch wenn einige zusammenwirkende Gesetzesstellen erst nach mehrfachem Lesen erkennbar sind. Wirkliche richterliche Freiheit ist nur bei der Bewertung bedenklicher Zeugenaussagen im § 211 erkennbar. Vielleicht erklärt sich das Misstrauen in eine frei urteilende Richterschaft auch aus dem absolutis-

\footnotetext{
${ }^{110}$ Dazu besonders für die österreichische Situation DAHLMANNS, Österreich 2723.

${ }^{111}$ HeINDL, Gehorsame Rebellen 101-125, 150, 235, 262-266; LAICH, Zwei Jahrhunderte Justiz 41-50, 311362.

${ }^{112}$ CANSTEIN, Civilprozessrecht 2, 279 i.V.m. CANSTEIN, Civilprozessrecht 1, 202.

113 SPRUNG, Grundlagen 386.
} 
tisch geprägten Geist der Zeit heraus und daher, dass der Richterstand primär den Beamten zugerechnet wurde und man ihm daher wohl auch aus dieser Perspektive keine allzu große Selbständigkeit in der Arbeit zutrauen wollte. Hierfür spricht auch, dass eine weitläufigere fundamentierte juristische Ausbildung des Beamtenstandes erst in der zweiten Hälfte des 18. Jahrhunderts begann. ${ }^{114}$ Der strenge Haftungsmaßstab den $\S 520$ WGGO normiert, um den Richter seine Grenzen aufzuzeigen, zeigt bereits das "Vertrauen" des Gesetzgebers in seine Richterschaft. Im Vergleich ergeben sich zwischen der WGGO und dem CIBI bei den Beweistheorien wenig Unterschiede. Bei beiden Kodifikationen wird die geringe Freiheit des Richters durch die Kommentarliteratur beschränkt. Die Prozessordnung in bürgerlichen Rechtsstreitigkeiten für das Königreich Bayern stellt ein beachtenswertes Mischsystem dar, dessen genauere Betrachtung auch den Erkenntnisstand heutiger zivilprozessualer Problemzonen und der mitteleuropäischen zivilprozessualen Entwicklungsgeschichte fördern könnte.

Geipel bringt in seiner Kritik an der heutigen Beweiswürdigung Folgendes auf den Punkt: „Der Rechtsstaat befindet sich - von der Wissenschaft weitgehend unbemerkt - in der Krise." Es mangelt an Untersuchungsergebnissen, die dem heutigen Revisionsverfahren ein gutes Zeugnis ausstellen und die Frage der Beweiswürdigung liegt völlig im Belieben des Richters und verortet eine hohe Dunkelziffer an Enttäuschten, die sich in die „innere Emigration“ zurückgezogen haben. ${ }^{115}$

${ }^{114}$ Vgl KITKA, Beweislehre im österreichischen CivilProcesse 1ff.; zur Juristenausbildung der Advocaten und über 6000 Richter zur Zeit der AGO vgl. MAYR, Praktische Juristenausbildung 114-117; KOCHER, Franz von Zeiller und seine Vorstellungen über eine Zivilprozessordnung 103.

${ }^{115}$ GEIPEL, Handbuch der Beweiswürdigung Kap. 14, Rz. 1, 8, 35 .
Der Vorgang, dem Richter die Beurteilung der Beweisergebnisse unmittelbar zu übertragen, ist auf der einen Seite ein Vertrauensbeweis des Staates in die von ihm ausgebildete Richterschaft die notwendigen Rückschlüsse in den für wahr zu haltenden Geschehensablauf aufgrund der eigenen Lebenserfahrung zu treffen, andererseits ein pragmatischer Grund zur Hintanhaltung ewiger Prozessreigen, da die Lebenserfahrung eines Menschen - außer in gravierenden Fällen - selbst wenn sie begründet wird, nicht ernsthaft rechtlicher Gegenargumentation ausgesetzt werden kann.

Die Beweismaßdiskussionen in den letzten Jahrzehnten des vorherigen Jahrhunderts zeigen aber, dass ein gewisser „Handlauf“ zur Überprüfung der getroffenen Feststellungen - wie ihn die legalen Beweistheorien vormals boten abgängig scheint. Ob vorrangig das Überwiegensprinzip oder die subjektive Beweismaßtheorie propagiert wird, hängt dabei vom aktuellen Standpunkt ab.116 Es ist ein stetiger Wechsel zwischen freier und gebundener Beweiswürdigung im Laufe kodifikatorischer Stufen auszumachen. Führte die freie Beweiswürdigung zu Willkür, wurden im Gegenzug wiederum feste Beweisregeln eingeführt, bis diese sich wieder mit der Vielschichtigkeit der Lebenssachverhalte spießten. ${ }^{117}$ Alle Versuche, den Richter mittels legaler Beweistheorien und -regeln in der Wahrheitsfindung „an die kurze Leine zu legen“, scheiterten jedoch. Die mündliche unmittelbare Verhandlung und Beweisaufnahme unter Einbeziehung der Öffentlichkeit, kein numerus clausus für Beweismittel, das rechtliche Gehör der Parteien, sowie die Begründungspflicht scheinen ausreichende Mittel zu sein, ein Vertrauen der Bevölkerung in die Justiz und in die richterlichen Handlungen zu gewährleisten und

\footnotetext{
116 ReCHBERGER, Maß für Maß 471-474.

117 GeIPEL, Handbuch der Beweiswürdigung Kap. 2, Rz. 22f; HubER, Beweismaß im Zivilprozeß 55.
} 
eine Willkür des Richters hintanzuhalten. ${ }^{118}$ Eine ernsthafte Bedrohung der freien Beweiswürdigung kann sicher nur in einer judikativen Aushöhlung und Nichtbeachtung anderer Verfahrensgrundsätze, beispielsweise der Wahrheitspflicht, ${ }^{119}$ geschehen. ${ }^{120}$ Im Interesse der Allgemeinheit sind Prozesse daher zu vermeiden und wenn notwendig, effizient und kostensparend unter der Leitung eines ungebundenen Richters als "Repräsentanten des Gemeininteresses" sowie als soziale „Betriebsunfälle“ zu einem schnellen, gütlichen Ende zu führen. Es darf der gesellschaftliche Nutzen des Prozesses nicht durch die Gestalt des Verfahrens verkümmern. ${ }^{121}$

Es bleibt daher lediglich in Erinnerung zu rufen, dass legale Beweistheorien zwar offensichtliche Prozesssicherheit hinsichtlich des Ablaufes generieren, aber diese an der besonders heute stärker vorwärtstreibenden Flexibilität der Lebenswirklichkeit ein jähes Ende finden können und mit dem eigentlichen legistischen Streben nach beständigen Kodifikationen - wie es das ABGB oder die ZPO vorleben - unvereinbar sind.

\section{Korrespondenz:}

Mag. Rainer Silbernagl

Universität Innsbruck

Institut für Römisches Recht und Rechtsgeschichte Innrain 52, 6020 Innsbruck

rainer.silbernagl@uibk.ac.at

\section{Abkürzungen:}

CIBI Codex Juris Bavarici Judiciarii

Siehe auch das allgemeine Abkürzungsverzeichnis: http://www.rechtsgeschichte.at/beitraege/abk.pdf

\footnotetext{
118 WALTER, Freie Beweiswürdigung 76.

${ }^{119}$ BREHM, Bindung des Richters 1, 57f.

${ }^{120}$ WALTER, Freie Beweiswürdigung 352f.

121 SPRUNG, Grundlagen 392-393.
} 


\section{Literatur:}

Heinz BARTA u.a. (Hgg.), Kontinuität im Wandel - 200 Jahre ABGB (1811-2011) (Innsbruck 2012).

Christian BALzER, Beweisaufnahme und Beweiswürdigung im Zivilprozess (Düsseldorf 2000).

Heinz BARTA, Karl KOHLEgGer, Viktoria StAdLMAyeR (Hgg.), Franz Gschnitzer Lesebuch 2 (Wien 2013).

Hermann BALTL, Gernot KOCHER, Österreichische Rechtsgeschichte (Graz $\left.{ }^{12} 2009\right)$.

Moritz BRINKMANN, Das Beweismaß im Zivilprozess aus rechtsvergleichender Sicht (München 2005)

Willhelm BRAUNEDER, Europäische Privatrechtsgeschichte (Wien-Köln-Weimar 2014).

Wolfgang BREHM, Die Bindung des Richters an den Parteienvortrag und Grenzen freier Verhandlungswürdigung (Tübingen 1982).

Raban CANSTEIN, Das Civilprozessrecht, 2 Teile (Berlin 1885).

Gerhard Dahlmanns, Die Josephinischen Prozeßordnungen und ihr System, in: Helmut COING (Hg.), Handbuch der Quellen und Literatur der neueren europäischen Privatrechtsgeschichte (München 1982) 2699-2754.

Heinrich Gottfried DANIELS, Code d'instruction criminelle aus dem franzöisischen nach dem oficiellen Texte übersetzt (Köln 1812)

Barbara DölemeYer, Heinz MoHnhaupt (Hgg.), 200 Jahre ABGB (1811-2011) Die österreichische Kodifikation im internationalen Kontext (Frankfurt am Main 2012).

Hans DolINAR, Richard HolZHAMMER, Zivilprozeßrecht I - Grundstudium (Freistadt ${ }^{52004) .}$

Willhelm EndEMAnN, Die Beweislehre des Civilprozesses (Heidelberg 1860)

Andreas Evers, Begriff und Bedeutung der Wahrscheinlichkeit für die richterliche Beweiswürdigung (Freiburg 1979).

Michael FORCHER, Bayern-Tirol Die Geschichte einer freud-leidvollen Nachbarschaft (Wien 1981).

Andreas GEIPEL, Handbuch der Beweiswürdigung (München 22013).

Waltraud HeINDL, Gehorsame Rebellen. Bürokratie und Beamte in Österreich Bd. 1: 1780-1848 (WienKöln-Weimar 2013).

Sebastian HiERETH, Die bayerische Gerichts- und Verwaltungsorganisation vom 13. bis 19. Jahrhundert. Einführung zum Verständnis der Karten und Texte (München 1950).

Michael HuBER, Das Beweismaß im Zivilprozeß, (München 1983).
Max KASER, Rolf KNÜTEL, Römisches Privatrecht (München 202014).

Rodrich KALIK, Die Beweiswürdigung im zivilgerichtlichen Verfahren, in: ÖJZ 7 (1954).

Joseph KITKA, Die Beweislehre im österreichsichen Civil-Processe (Wien 1842).

Michael KoBLER, Bayerische Kodifikationen des Naturrechtszeitalters, in: HRG ${ }^{1}$ (Berlin 1971) 337-339

Michael KOBLER, Bayern, in: HRG ${ }^{1}$ (Berlin 1971) 331337.

Georg KoDEK, Peter MAYR, Zivilprozessrecht (Wien 22013)

Gernot KocHer, Franz von Zeiller und seine Vorstellungen über eine Zivilprozessordnung, in: Daphne-Ariane SimotTA (Hg.), Der Zivilprozess zu Beginn des 21. Jahrhunderts Vergangenheit, Gegenwart und Perspektiven. FS für Wolfgang Jelinek zum 60. Geburtstag (Wien 2002) 101 - 109.

Karl Kroeschell, Deutsche Rechtsgeschichte, Bd. 3 (Köln-Weimar-Wien ${ }^{52005) . ~}$

Wiguläus KREITTMAYR, Anmerkungen über den Codex juris Bavarici judiciarii: worinn derselbe sowohl mit den gemeinen, als den ehmaligen statutarischen Gerichts-Ordnungen und Rechten genau verglichen ist (München 1813).

Mario LAICH, Zwei Jahrhunderte Justiz in Tirol und Vorarlberg (Innsbruck-Bozen 1990).

Michael LOSCHELDER, Die österreichische Allgemeine Gerichtsordnung von 1781 Grundlagen und Kodifikationsgeschichte (Berlin 1978).

Peter MAYR, Die praktische Juristenausbildung im Zeitalter des ABGB in Heinz BARTA u.a. (Hgg.), Kontinuität im Wandel. 200 Jahre ABGB (18112011) (Innsbruck 2012).

Anton MENGER, System des österreichischen Zivilprozesses in rechtsvergleichender Darstellung (Wien 1876).

Heinrich MitTeIs, Heinz LiEBERICH, Deutsches Privatrecht (München ${ }^{9} 1981$ ).

Erich Molitor, Hans Schlosser, Grundzüge der neueren Privatrechtsgeschichte (Karlsruhe ${ }^{2} 1975$ ).

Monika Niedermayr, Rainer OBERHUMMER, Clemens PEER, Ratsprotokolle der Obersten Justizstelle, in: Heinz BARTA u.a. (Hgg.), Kontinuität im Wandel 200 Jahre ABGB (1811-2011) (Innsbruck 2012).

Franz MATSCHER, Die Entwicklung des zivilprozessualen Rechts, in: Herbert SCHAMBECK (Hg.), Parlamentarismus und öffentliches Recht in Österreich Entwicklung und Gegenwartsprobleme, Teilbd. 1 (Berlin 1993) 475-518.

Hans-Joachim MusIELAK, Max STADLER, Grundfragen des Beweisrechtes (München 1984). 
Franz Xaver NIPPEL, Erläuterung der Allgemeinen Gerichtsordnung vom 1. Mai 1781 nebst einem Anhange die Erörterung der Abweichungen der Westgalizischen Gerichtsordnung enthaltend, Bd. 1 (Wien 1845).

Paul OBERHAMMER, Zu den Ursprüngen des Mahnverfahrens im österreichischen Recht, in: Bernhard KÖNIG (Hg.), Historiarum ignari semper sunt pueri. FS Rainer Sprung zum 65. Geburtstag (Wien 2001) 283-310.

Werner OGRIS, Die Rechtsentwicklung in Cisleithanien 1848-1918, in: Adam WANDRUSZKA, Peter URBANITSCH (Hgg.), Die Habsburgermonarchie 1848-1918, Bd. II: Verwaltung und Rechtswesen (Wien 1975) 538-662

Carl Joseph Pratobevera, Ueber den Beweis durch Zeugen im Oesterreichischen Civil-Processe in: Materialien für Gesetzeskunde und Rechtspflege in den Osterreichischen Staaten 7 (1823) 199-367.

Arthur RASSE, Die Entwicklung der Juristenausbildung und der juristischen Berufsprüfungen in Österreich seit dem 18. Jahrhundert (Wien 2012).

Johannes Michael RAINER, Vom Probebetrieb zum 200-jährigen Bestehen, in: Bundesministerium für Justiz (Hg.), 200 Jahre ABGB. RichterInnenwoche 2011 in Lochau, 16.-20. Mai 2011 (Wien-Graz 2012) 19-26.

Walter RECHBERGER, Daphne-Ariane SiMOTTA, Grundriss des österreichischen Zivilprozessrechts. Erkenntnisverfahren (Wien ${ }^{82010) .}$

Walter ReCHBERGER, Maß für Maß im Zivilprozeß? Ein Beitrag zur Beweißmaßdiskussion, in: Hanns PRÜTTING (Hg.), Festschrift für Gottfried BaumGärtel (Köln 1990).

Egon SCHNEIDER, Beweis und Beweiswürdigung (München ${ }^{41987) . ~}$

Hans SCHLOSSER, Grundzüge der neueren Privatrechtsgeschichte (Heidelberg ${ }^{102005)}$.

Hans SCHLOSSER, Spätmittelalterlicher Zivilprozess nach bayerischen Quellen (München 1971).
Martin SCHENNACH, ABGB - kodifikatorisches Umfeld (im Erscheinen)

Martin SCHENNACH, Revolte in der Region: zur Tiroler Erhebung von 1809 (Innsbruck 2009).

Johann SEIDL, Allgemeine Taxnorme für alle Gegenstände obrigkeitlicher und richterlicher Amtshandlungen (Prag 1802).

Hans SPERL, Lehrbuch der Bürgerlichen Rechtspflege, Bd. 1, Teil 1 (Wien 1925)

Rainer SPRUNG, Das zivilgerichtliche Verfahren, in: Gerald Prabitz, Wolfgang Schopper (Hgg.), Uni 2000 - Zukunftsperspektiven universitärer Lehre und Forschung am Beispiel der Universität Innsbruck (Innsbruck 1990) 146-151.

Rainer SPRUNG, Die Entstehung und Weiterentwicklung des Außerstreitgesetzes 1854, in: Bundesministerium für Justiz (Hg.), Ausserstreitverfahren. Die fällige Reform (Wien 1996) 11-60.

Rainer SPRUNG, Die Grundlagen des österreichischen Zivilprozeßrechts, in: Zeitschrift für Zivilprozeß, 90 (1977) 380-394.

Moriz StubenRauch, Systematisches Handbuch der Literatur der allgemeinen (josephinischen) Gerichtsordnung vom 1. Mai 1781 der westgalizischen Gerichtsordnung vom 19. December 1796, der italienischen Gerichtsordnung vom 16. März 1803, der tyrolischen Gerichtsordnung vom 15. September 1814 und der allgemeinen josephinischen Concursordnung v. 1. Mai 1781 (Wien 1840).

Otto TschadeK, Der Beweis. Eine Betrachtung über Beweismittel und Beweiswürdigung (Wien 1958).

Gerhard WALTER, Freie Beweiswürdigung (Tübingen 1979).

Gerhard WesenBerg, Gunter WeSENER, Neuere deutsche Privatrechtsgeschichte (Graz-Wien ${ }^{4} 1985$ ).

J. WERnZ, Commentar zur Prozeßordnung in bürgerlichen Rechtsstreitigkeiten für das Königreich Bayern, erste Abtheilung (München 1871). 